Ruben J. Chua Jr. MD

Department of Otolaryngology

Head and Neck Surgery

Amang Rodriquez Memorial Medical Center
Rene C. Lacanilao MD

\section{Facial Paralysis in Longitudinal versus Oblique and Otic-Sparing versus Non Otic-Sparing Temporal Bone Fractures}

\begin{abstract}
Objective: To compare the proportion of temporal bone fractures using traditional (longitudinal vs. transverse) and otic involvement (otic sparing vs. non-otic sparing) classification schemes and their relationship with the development of facial paralysis.
\end{abstract}

Methods:
Design:
Retrospective Case Series
Setting:
Tertiary Government Hospital
Participants: Records of 49 patients diagnosed with temporal bone fracture in our institution from August 2016 to June 2018.

Results: A total of 41 records of patients with temporal bone fractures, 32 males, 9 females, aged 5 to 70 years old (mean 37.5 years old) were included. In terms of laterality, 23 (56\%) involved the right and 17 (41\%) the left side. Traditionally classified, 32 (78\%) were longitudinal and 9 (22\%) were transverse. Using newer classification based on otic involvement and non-otic involvement, $38(93 \%)$ were otic-sparing and $3(7 \%)$ were non otic-sparing. Only $9(22 \%)$ out of 41 total fracture patients developed facial paralysis, involving 7 of the 32 longitudinal fractures and 2 of the 9 transverse fractures, or 8 of the 38 otic-sparing and 1 out of 3 non otic-sparing fractures.

Department of Otolaryngology-Head and Neck Surgery

Amang Rodriguez Memorial Medical Center

Sumulong Highway, Sto. Nino, Marikina 1800

Philippines

Phone: (632) 89415854

Email: armmc_orlhns@yahoo.com

The authors declare that this represents original material, that the manuscript has been read and approved by all the authors, that the requirements for authorship have been met by each author, and that the authors believe that the manuscript represents honest work.

Disclosures: The authors signed a disclosure that there are no financial or other (including personal) relationships, intellectual passion, political or religious beliefs, and institutional affiliations that might lead to a conflict of interest.

Presented at the Philippine Society of Otolaryngology Head and Neck Surgery Descriptive Research Contest. October 22, 2018, Maynila Ballroom, The Manila Hotel, Manila. accidents; facial paralysis

Temporal bone fractures have been traditionally classified according to the fracture plane described as longitudinal or transverse in relation to the petrous ridge. A more recent classification gaining popularity describes fractures in terms of whether they penetrate the bony labyrinth (i.e. the cochlea, vestibule or semi-circular canals) as otic-capsule violating; or not, classified as oticcapsule sparing. ${ }^{1}$ The change in classification scheme allows a focus on the functional sequelae 
and complications of temporal bone fractures as opposed to merely describing the anatomic orientation of the fracture. ${ }^{2}$

For example, a study of 173 patients done in Korea found otic capsule-sparing fractures in 188 temporal bones by high-resolution computed tomography (CT). Of these, $128(68 \%)$ were longitudinal, $23(12 \%)$ were transverse and 37 (20\%) were mixed. Among the 188 fractures there were 17 cases of facial paralysis (9\%). Out of these 17 , 10 were longitudinal (7.8\% of the 128 longitudinal fractures), 3 were transverse (13.0\% of the 23 transverse fractures) and 4 were mixed types (10.8\% of the 37). ${ }^{3} \mathrm{~A}$ recent retrospective review showed that the otic capsule-sparing versus otic capsule-disrupting classification scheme demonstrated statistically significant predictive ability of determining facial nerve paralysis when compared with the older classification scheme. ${ }^{3}$

This study sought to compare the proportion of temporal bone fractures using traditional (longitudinal vs. transverse) and otic involvement (otic sparing vs. non-otic sparing) classification schemes and their relationship with the development of facial paralysis.

\section{METHODS}

With Institutional Ethical Review Board approval (ERB No. R-201805-00), this retrospective case series retrieved records of patients with temporal bone fractures seen in our institution from August 2016 to June 2018. Potential cases were identified from admission, referral and emergency room logbooks and the daily in-patient census.

Included were records of those seen in or referred to the emergency room department and in-patient ward with a suggestive history and documented symptoms and signs (hemotympanum, bloody otorrhea, battle sign) of temporal bone fracture, and CT scan diagnoses of temporal bone fracture that had been made by a senior radiology resident. Diagnoses of facial paralysis had been previously recorded in the charts by an ear, nose, throat (ENT) resident physician who performed the physical examination, using the House-Brackmann classification.

The hospital Picture Archiving and Communication System (PACS) was utilized to retrieve Digital Imaging and Communications in Medicine (DICOM) images of CT scans that had been obtained using a HITACHI ECLOS 8 CT Scanner (Hitachi Medical Systems Europe AG, Steinhausen, Switzerland). Retrieved DICOM images were independently reviewed by a blinded senior radiology resident and only those diagnosed with temporal bone fractures were included in the final analysis.

Excluded were records of patients with previous facial paralysis or asymmetry, those whose $\mathrm{CT}$ scan images could not be retrieved on our PACS, and those with signs or symptoms of temporal bone fracture that were not confirmed by $\mathrm{CT}$ scan, or when no temporal bone fractures could be confirmed upon review by the senior radiology resident.

Data from patient records was tabulated using MS Excel version 14.6.3 (Microsoft Corp., Redmond, WA, USA) and variables were analyzed using descriptive statistics (frequencies and percentages).

\section{RESULTS}

Out of a total of 49 patients identified, only 41 patients with temporal bone fractures were included in this series, aged 5 to 70 years old (mean age 37.5 years old). Thirty two $(78.05 \%)$ were male and $9(21.96 \%)$ were female. Of the 41 fractures, 32 (78.05\%) were longitudinal, 9 (21.96\%) were transverse, 38 (92.68\%) were otic-sparing and $3(7.32 \%)$ were non otic-sparing. Based on laterality $23(56.10 \%)$ occurred in the right side and 17 (41.46\%) occurred in the left. Figures 1 to 4 show representative CT scan images of longitudinal, transverse, otic-sparing and non oticsparing fractures.

Out of 32 longitudinal temporal bone fractures, $7(21.88 \%)$ had facial paralysis. Out of 9 transverse temporal bone fractures, 2 (22.22\%) had facial paralysis. On the other hand, out of 38 otic-sparing temporal bone fractures, 8 (21.05\%) had facial paralysis and out of 3 otic-disrupting bone fractures only 1 (33.33\%) had facial paralysis. In other words, facial paralysis occurred on the average in $22 \%$ of all temporal bone fractures whether or not they were longitudinal fractures, transverse fractures, or otic-sparing fractures, while otic-disrupting fractures had a facial paralysis occurrence of $33 \%$.

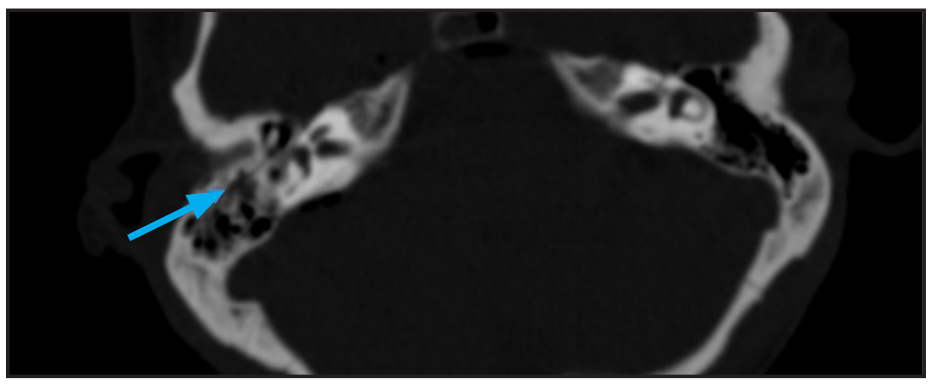

Figure 1. Representative plain temporal bone CT Scan, axial view, bone window at the level of the mastoid air cells showing a longitudinal fracture (arrow).

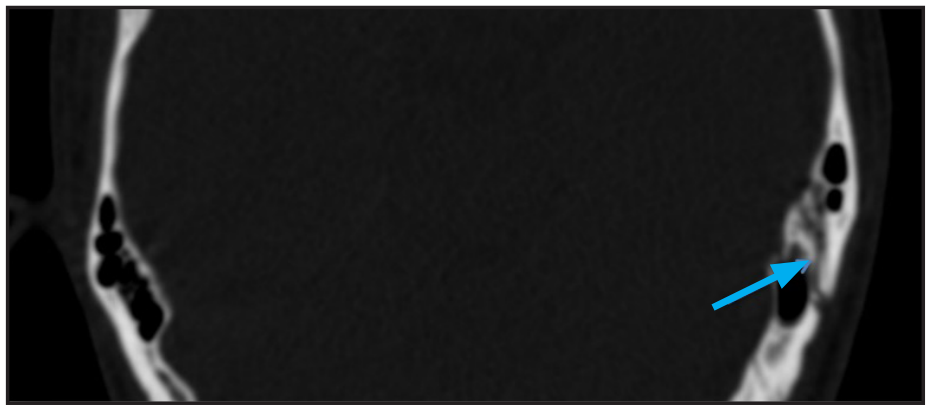

Figure 2. Representative plain temporal bone CT Scan, coronal view, bone window at the level of the mastoid air cells showing a transverse fracture (arrow). 


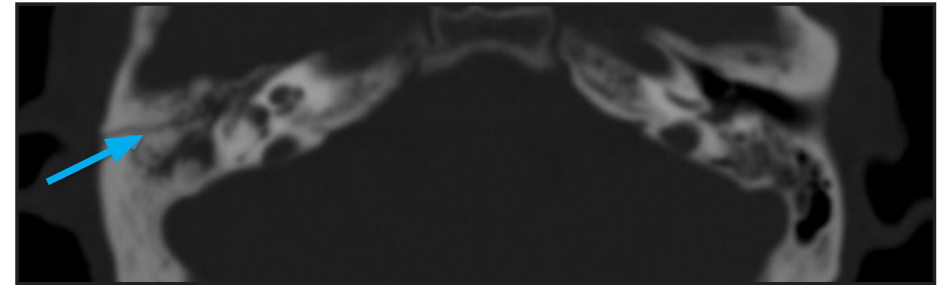

Figure 3. Representative plain temporal bone CT Scan, axial view, bone window at the level of the otic capsule showing an otic-sparing fracture (arrow).

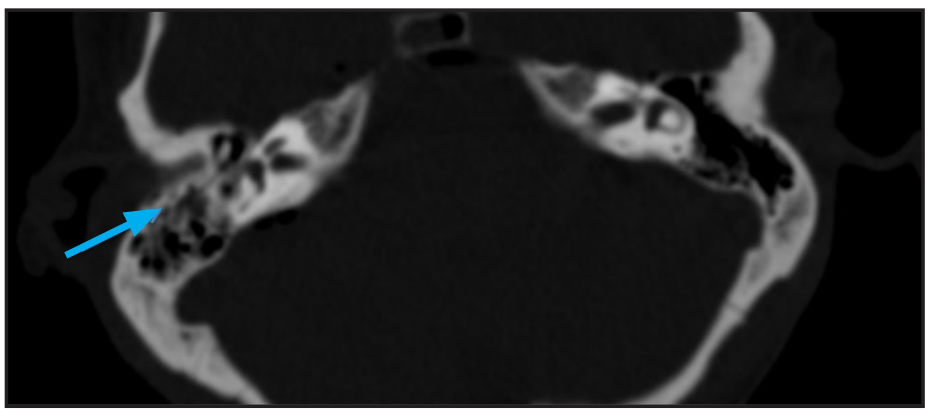

Figure 4. Representative plain temporal bone CT Scan, axial view, bone window at the level of the otic capsule showing an otic-disrupting fracture (arrow).

\section{DISCUSSION}

Our study found that the percentage of facial paralysis was on the average $22 \%$ regardless of the type of fracture. There was no significant difference in proportion of facial paralysis when classified according to the traditional or newer classification scheme for temporal bone fractures. Although otic-disrupting fractures had a facial paralysis occurrence of $33 \%$, it is hard to draw a conclusion on their occurrence and relationship to facial paralysis because of the small sample size.

Facial paralysis is a severely disfiguring complication of temporal bone fractures wherein $7 \%$ result in facial paralysis. ${ }^{2}$ Facial nerve palsy occurs in $20 \%$ of longitudinal temporal bone fractures and $50 \%$ of transverse temporal bone fractures. ${ }^{4} \mathrm{~A}$ previous study found that the incidence of facial paralysis was 10\%-20\% in longitudinal fractures and $50 \%$ in transverse fractures. ${ }^{5}$ Both longitudinal and oblique fractures run parallel to the long axis of the petrous ridge and together account for $75-80 \%$ of temporal bone fractures with the facial nerve involved in $20-25 \% .{ }^{6}$ A study by Proctor et al. demonstrated that $80 \%$ of temporal bone fractures were longitudinal and 20\% were transverse. ${ }^{7}$ Several studies shows that $50 \%$ of transverse fractures will present with facial nerve paralysis. ${ }^{8,9}$

Although the results of this study mirrored the observation that the anatomical classification of temporal bone fractures into longitudinal and transverse types may be convenient but offer no clinical distinction in predicting the development of facial nerve paralysis, the limitations of our study need to be considered.
A primary limitation of this study is the small sample size, especially reflected in the otic-disrupting subset that is not large enough to compare the occurrence of facial nerve paralysis. Future studies with larger samples may yield better results for analysis.

In conclusion, because of the small sample size, this study can draw no conclusions regarding the proportion of temporal bone fractures using traditional (longitudinal vs. transverse) and otic involvement (otic sparing vs. non-otic sparing) classification schemes and their relationship with the development of facial paralysis.

\section{REFERENCES}

1. Bhindi A, Carpineta L, Al Qassabi B, Waissbluth S, Ywakim R, Manoukian JJ, et al. Hearing loss in pediatric temporal bone fractures: Evaluating two radiographic classification systems as prognosticators . Int J Pediatr Otorhinolaryngol. 2018 Jun; 109: 158-163. DOI: 10.1016/j. ijporl.2018.04.005; PMID: 29728172.

2. Brodie $\mathrm{H}$, Wilkerson B. Management of Temporal Bone Fracture. In: Flint PW, Haughey BH, Lund V, Niparko JK, Thomas Robbins K, Regan Thomas J, et al. Cumming's Otorhinolaryngology- Head and Neck Surgery. 6th edition. PA: Elsevier Saunders. 2015. pp. 2220-2232.

3. Song SW, Jun BC, Kim H. Clinical features and radiological evaluation of otic capsule sparing temporal bone fractures. J Laryngol Otol. 2017 Mar; 131(3): 209-214. DOI: 10.1017/ S0022215117000123; PMID: 28124635

4. Stewart MG. Head, Face, and Neck Trauma. $1^{\text {st }}$ edition.. NY: Thieme New York. 2005. pp. 170 172.

5. Nadol Jr., JB, McKenna MJ. Surgery of the Ear and Temporal Bone. PA: Lippincott Williams and Wilkins. 2005. pp. $442-447$.

6. Schubl SD, Klein TR, Robitsek RJ, Trepeta S, Fretwell K, Seidmann D, et al. Temporal bone fracture: Evaluation in the era of modern computed tomography. Injury. 2016; 1893-7. DOI: 10.1016/j.injury.2016.06.026; PMID: 27387791.

7. Grewal DS, Hathiram BT. Facial nerve in temporal bone fractures: In: House W, (editor). Atlas of surgery of the facial nerve. Philadelphia: McGraw-Hill .2007. pp. 50-57.

8. Dela Cruz RR, Tuazon R. Motorcycle related cranio-maxillofacial injuries at a tertiary hospital in the Philippines. Philipp J Otolaryngol Head Neck Surg. 2016 Jul-Dec; 31(2): 27-30. DOI: https://doi. org/10.32412/pjohns.231.

9. Yalciner G, Kutluhan A, Bozdemir K, Cetin H, Tarlak B, Bilgen AS. Temporal bone fractures: evaluation of 77 patients and a management algorithm. Turkish Journal of Trauma \& Emergency Surgery. 2012 Sep; 18(5): 424-428. DOI: 10.5505/tjtes.2012.98957; PMID: 23188604 